\section{DOES THE CORRECTED TIMI FRAME COUNT ACTUALLY REPRESENT CORONARY FLOW AND MICROVASCULAR RESISTANCE?}

Bhavya Pydi, Mesk Alsubaiei*, Matthew Ryan, Haseeb Rahman, Divaka Perera. King's College London

\subsection{6/heartjnl-2019-BCS.68}

Introduction Corrected Thrombolysis in myocardial infarction Frame Count (CTFC) is a key determinant of several emerging techniques aimed at estimating coronary stenosis severity, such as QFR. However, the relationship between CTFC and coronary flow or microvascular resistance (MR) has not been clearly established. This study aimed to assess the correlation between CTFC and coronary physiological indices.

Methods Simultaneous intra-coronary pressure and flow velocity were acquired using a Combowire (Philips, Volcano) in the LAD vessel of patients with angina, non-obstructive coronary artery disease (Fractional Flow Reserve (FFR) $>0.80$ ) and preserved left ventricular systolic function. CTFC was measured by blinded observers, as the number of frames taken for contrast to reach a fixed landmark in the distal LAD. MR was calculated as distal coronary pressure/APV.

Results 64 patients were studied (80\% Female, Age $57 \pm 10$, $57 \%$ HTN, 23\% DM, 29\% Smokers) with mean $( \pm$ SD) FFR $0.92 \pm 0.04$, CFR $2.37 \pm 0.66$. The mean CTFC and APV were 24 frames (SD, 9; range, 10-49) and $18.64 \mathrm{~cm} \cdot \mathrm{s}-1$ (SD, 6.11; range, 35.60-7.95), respectively. The mean $\mathrm{MR}$ was found to be 5.94 mmHg.s.cm-1 (SD, 2.04; range, 2.6210.85). There was a negligibly weak negative correlation between CTCF and APV $\left(r=0.25, \mathrm{r}^{2}=0.064\right)$ and no correlation between CTCF and MR $\left(r=0.055, r^{2}=0.003\right)$. Scatter plots of CTFC against $\mathrm{APV}$ and $\mathrm{MR}$ are shown in figures 1 and 2.

Conclusion This study demonstrates that visual angiographic estimations of coronary blood flow correlate poorly with directly measured values. Currently, measurements of coronary physiology during the time of angiography require the use of intra-coronary pressure and/or flow wires. These findings may explain the discordance between stenosis severity indices based on angiographic contrast clearance rates (such as QFR) and invasively derived measures such as FFR.

Conflict of Interest None

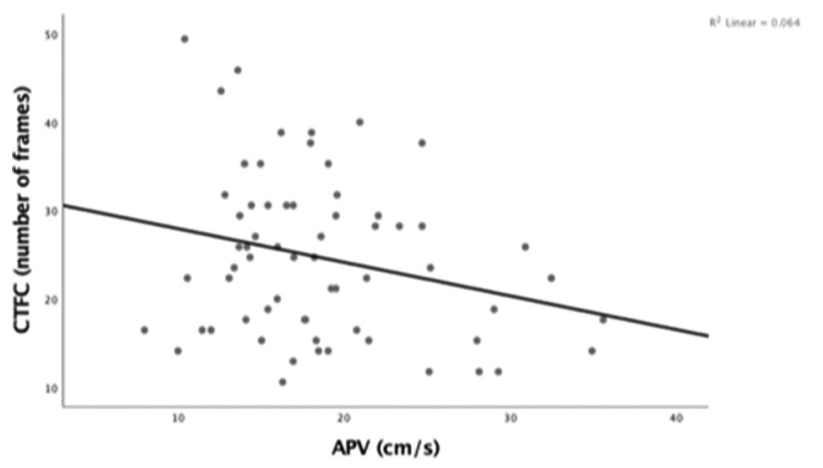

Abstract 70 Figure 1 Relation between CTFC and APV, n 64 measurements

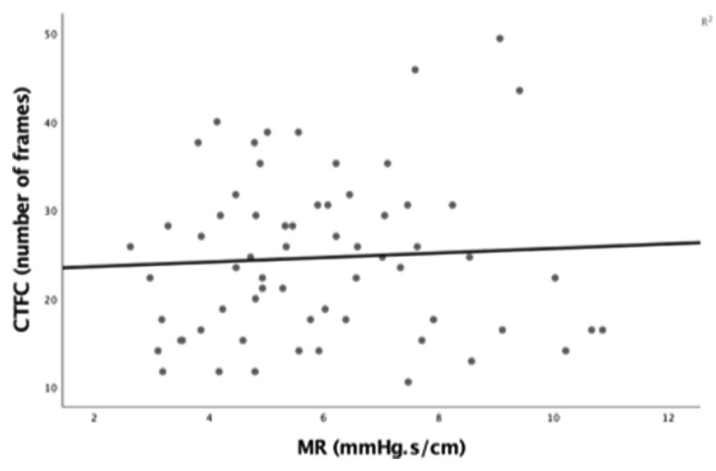

Abstract 70 Figure 2 Relation between CTFC and MR, n 64 measurements

\section{OUTCOME IN PATIENTS UNDERGOING HIGH-RISK PCI USING IMPELLA CIRCULATORY SUPPORT - 10 YEAR EXPERIENCE}

Vincenzo Vetrugno*, Muhammad Waqas, Kulwinder Sandhu, Anthony Mechery, M. Adnan Nadir, Sudhakar George, Alexander Zaphiriou, Sern Lim, Peter Ludman, Sagar Doshi, Jonathan Townend, sohail khan. Queen Elizabeth Hospital - Birmingham

\subsection{6/heartjnl-2019-BCS.69}

Introduction The use of Impella in the setting of high-risk percutaneous coronary intervention (PCI) has been introduced in clinical practice in the last few years in selected hospitals, giving a chance for revascularization to a sensible number of patients who are usually unsuitable for cardiac surgery due to high risks. In addition, some of these patients present pre-procedural cardiogenic shock, further increasing the rate of morbidity and mortality. Our purpose was to assess outcome of patients undergoing Impella-assisted high-risk PCI in our 10 year experience and to assess possible predictors of adverse events.

Methods From May 2008 to September 2018 patients undergoing Impella-assisted high-risk PCI were enrolled. Clinical, laboratory, echocardiographic, angiographic and procedural data were collected. Coronary artery disease burden was graded using the British Cardiovascular Intervention Society Jeopardy Score (BCIS-JS). In-hospital MACCE were recorded. During routine follow-up visits data including MACCE, hospital admissions for heart failure, Canadian Cardiovascular Society (CCS) angina grade and New York Heart Association (NYHA) functional class were recorded. Long-term survival Kaplan Meier analysis was performed according to national registry death data.

Results A total of 80 consecutive patients were enrolled (71.2 \pm 13.7 years, male gender 73.8\%), 21 (26.3\%) presenting with stable angina, $53(66.3 \%)$ with NSTE-ACS and $6(7.5 \%)$ with STEMI. $67(83.8 \%)$ of them showed multivessel disease, $42(52.5 \%)$ unprotected left main disease, 47 (58.8\%) severe left ventricle systolic dysfunction (LVEF<30\%), 10 (12.5\%) pre-procedural cardiogenic shock. Median BCIS-JS was 10 $[8,00 ; 12,00]$. In-hospital MACCE occurred in $16(20 \%)$ patients with death in $15(18.8 \%)$. Median time to first follow-up visit for survivors was $105(64.5 ; 282.0)$ days: at this time $11(13.8 \%)$ patients had MACCE, 3 (3.8\%) had hospital admissions for heart failure, median CCS was $0.00 \quad(0.00$; $0.00)$ and median NYHA was $1.00(1.00 ; 2.00)$. Mean survival time (procedure to death, months) was 21 months (C.I. 14.4 - 29.0). Multivariate logistic regression analysis for possible predictors of in-hospital MACCE was performed including 